\title{
Detection and susceptibility measurements of a single Dynal bead
}

Lorenzo Di Michele,1 Connor Shelly,1 Patrizia de Marco,1,2 Patrick See,1 David Cox,1

and Olga Kazakova1,a)

1National Physical Laboratory, Teddington, TW11 0LW, United Kingdom

${ }_{2}$ Universita' degli Studi dell'Aquila, L'Aquila, 67100, Italy

In this work we present detection and susceptibility measurement experiments on a single superparamagnetic Dynal bead with a diameter of $1 \mathrm{~lm}$ and a magnetic moment of _ 4 _ 108lB. Accurate bead positioning was achieved via non-invasive AFM nanomanipulation. The detection and magnetic characterization of the bead were performed using ultra-sensitive InSb Hall devices. Single bead detection was demonstrated using a step-wise change of the dc magnetic field; measurements were performed using only the in-phase component of the total ac Hall voltage. Very clear evidence of the bead presence is demonstrated simultaneously with explicit separation of parasitic inductive signals. Additional experiments performed using a sweeping change of the dc field allowed susceptibility measurements of a single Dynal bead. The numerical outcomes of both sweeping and stepping experiments are in a very good agreement. The method presented here opens up new possibilities for the reliable and accurate detection of small magnetic moments, which is of high importance for metrological applications as well as highly sensitive biological, medical, and environmental detectors. 


\section{INTRODUCTION}

For decades, Hall sensors have continued to be one of the most important types of magnetic field sensors, with numerous applications in the automotive, consumer electronics, communications, industrial, aerospace, and defense markets. The low price and high reliability of Hall sensors sustain their market growth. During the past year, the usage of Hall sensors in cell phones, gaming, and other consumer electronic products led to a significant increase in their production volume. As a result, Hall sensors are expected to continue to dominate the world magnetic sensors market. The predicted revenue and revenue growth rate for the Hall sensor market in 2016 are $\$ 2,204 \mathrm{M}$ and $9.6 \%$, respectively. 1

Recently, miniaturized Hall sensors came to occupy a large niche in biomolecular and nanomedical applications.2-4 Such sensors combine very good field sensitivity, easy integration with electronic devices, and high performance at room temperature. Both industrial and research applications require sensors with decreasing dimensions, very often in the submicron range. Although scaling down the lateral dimensions of Hall sensors causes an increase of the voltage noise, 5 the general sensitivity of such sensors is still sufficient for the detection of individual micron and nanosized magnetic beads, $6-11$ as required for biological, medical, and environmental applications. A general trend toward the miniaturization of both sensors and magnetic labels (beads) leads to a fundamental challenge of the reliable detection of a true magnetic signal and its separation from parasitic signals (for example, signals generated by inductive couplings, foreign ferromagnetic materials in the vicinity of the device, etc.). In our previous research we demonstrated a further development of the ac-dc Hall magnetometry technique (initially proposed by Besse et al.6) based on the measurement of the in-phase component of ac Hall voltage. Using this method, we showed that such separation of the real and parasitic signals is indeed possible, even on a very small scale. The detection of a FePt nanobead with a size of $140 \mathrm{~nm}$ and a moment of 108 lB was successfully performed at room temperature using a sweeping dc field method.12 Although in our earlier work we demonstrated the detection of one of the smallest single magnetic particles ever reported in the literature, the coupling between the particle and the sensor has remained far from ideal (C . $\left.4 \_10 \_5\right)$, and this has notably limited the sensitivity of the method. 
In the present paper we consider the case of significantly better coupling between the magnetic bead and the Hall sensor. We present both detection and susceptibility measurement experiments for a single superparamagnetic particle (Dynal bead) with a diameter of $1 \mathrm{~lm}$ and a magnetic moment of _ $4{ }_{-} 108 \mathrm{lB}$ using InSb double Hall crosses with a sensor area of $1 \mathrm{~lm} 2$. The coupling constant C.0.09 was numerically computed following Ref. 13. The coupling constant is the bead-sensor coupling coefficient, which quantifies how efficiently the sensor converts the bead's stray magnetic field into the Hall voltage. Accurate particle positioning has been achieved via contact mode AFM nanomanipulation. Optimization of the sensor working parameters was performed. Very clear evidence of the bead's presence $=$ absence has been demonstrated by the detection of the in-phase ac Hall voltage using an optimized ac-dc experimental setup and applying a step-wise dc magnetic field. Thus, the improved ac-dc method demonstrated here provides straightforward and unambiguous detection of the bead and a clear separation of the real and inductive signals, which is essential for applications. Additionally, we show that the Hall sensor is capable of susceptibility measurements of a single magnetic bead.

\section{METHODS}

\section{A. Sample fabrication}

Undoped InSb films about $300 \mathrm{~nm}$ thick were grown via two-phase molecular-beam epitaxy on semi-insulating GaAs (001) substrates at a base pressure of 10_10 mbar. A high electron mobility of $1.1: 3 \mathrm{~m}_{2}=\mathrm{Vs}$ and concentration of $\mathrm{n} .3: 9{ }_{-} 1016 \mathrm{~cm}_{-} 3$ were deduced from magnetoresistance measurements in a perpendicular magnetic field in the van der Pauw geometry. ${ }_{14}$ The InSb film was patterned into a double Hall cross geometry via electron beam lithography and reactive ion etching. Each sample was constructed of two symmetric crosses connected via the current lead. When a particle was present on one of the crosses, the second one was left empty as a control device. A constant 10:1 and 5:1 length to width aspect ratio was adopted for the central Hall bar region and the arms, respectively. The width of the Hall bar was $1 \mathrm{~lm}$. Ohmic contacts were formed by a non-alloyed evaporated titanium=gold layer on the samples. These metal contacts were spanned onto the bond pad areas and also along the mesa leads up to the edge of the double Hall cross arms.

Two-terminal linear Ohmic current-voltage resistances of _40 kX were recorded for all Hall bars, as expected for the constant length to width aspect ratio. This resistance was 
lower compared to previous values of $60 \mathrm{kX}$ due to the optimized Ohmic contact extents. The four-terminal resistance was _7 kX (for a total of 20 squares, i.e., 10:1 current bar plus two $5: 1 \mathrm{arms}$ ), giving $\_350 \mathrm{X}=$ square $\left(\mathrm{cf}_{-} 410 \mathrm{X}=\right.$ square estimated from the mobility and carrier concentration values by van der Pauw measurements14).

\section{B. Bead nanomanipulation}

Commercially available microbeads with a diameter of $1 \mathrm{~lm}$ (Dynal, MyOne) were used for the detection experiment, and similar $2.7 \mathrm{~lm}$ beads were used for transmission electron microscopy (TEM) imaging. Each bead contains nanometer sized ferrite particles embedded in a polymer matrix. The whole bead is covered by a monolayer of streptavidin, potentially providing a strong attachment to biotin labeled biomolecules. Figure 1(a) shows a TEM image of the cross-section of the $2.7 \mathrm{~lm}$ Dynal bead. The image was obtained by slicing a $\_100 \mathrm{~nm}$ thick section of the bead and imaging the resulting foil at $30 \mathrm{kV}$. For TEM imaging, a backscatter detection in the forescatter position was used. The main image in Fig. 1(a) shows the full cross-section of the bead in the dark field; the ferrite nanopartcles appear as bright spots on the dark background of the polymer matrix. The bright vertical streaks are areas of an inhomogeneous membrane thickness (i.e., too thick for the electron beam). A magnified section of the bead in which the nanoparticle contrast was artificially enhanced is shown in the inset of Fig. 1(a). The TEM image enables a direct estimate of the total number of ferrite nanoparticles, which is _ 106 per one-micron bead. This estimation was performed by evaluating the nanoparticle density in the foil. Magnetization measurements of the beads were carried out using a superconducting quantum interference device magnetometer (MPMS XL, Quantum Design) at room temperature and in fields of up to $2 \mathrm{~T}$ (Fig. 1(b)). The magnetic susceptibility decreases quickly with the dc magnetic field: $\mathrm{v}_{-} 0$ at $\mathrm{BDC}_{-} 0: 1 \mathrm{~T}$ (Fig. 1(b), inset). For single bead manipulation, a low-density droplet of beads dispersed in the stabilization buffer was deposited on the sensor substrate and left to dry. A Veeco Dimension Icon Scanning Probe Microscope equipped with NanoMan VS User Interface software was used for the imaging and positioning of a single bead onto the sensor. A single crystal silicon tip (NTMDT NSG01S) with a typical radius of 6 to $10 \mathrm{~nm}$, a frequency range of $87-230 \mathrm{kHz}$, and a spring constant range of 1.5-15.1 N=m was used to push the selected bead into the final position. The nanomanipulation process was performed in two steps. In the first step, the topography of the sample was acquired in tapping mode. In the second step, the tip 
moved following a predrawn path - a straight line - as indicated by the blue arrows in Fig. 2, with a well-established velocity and distance from the surface.

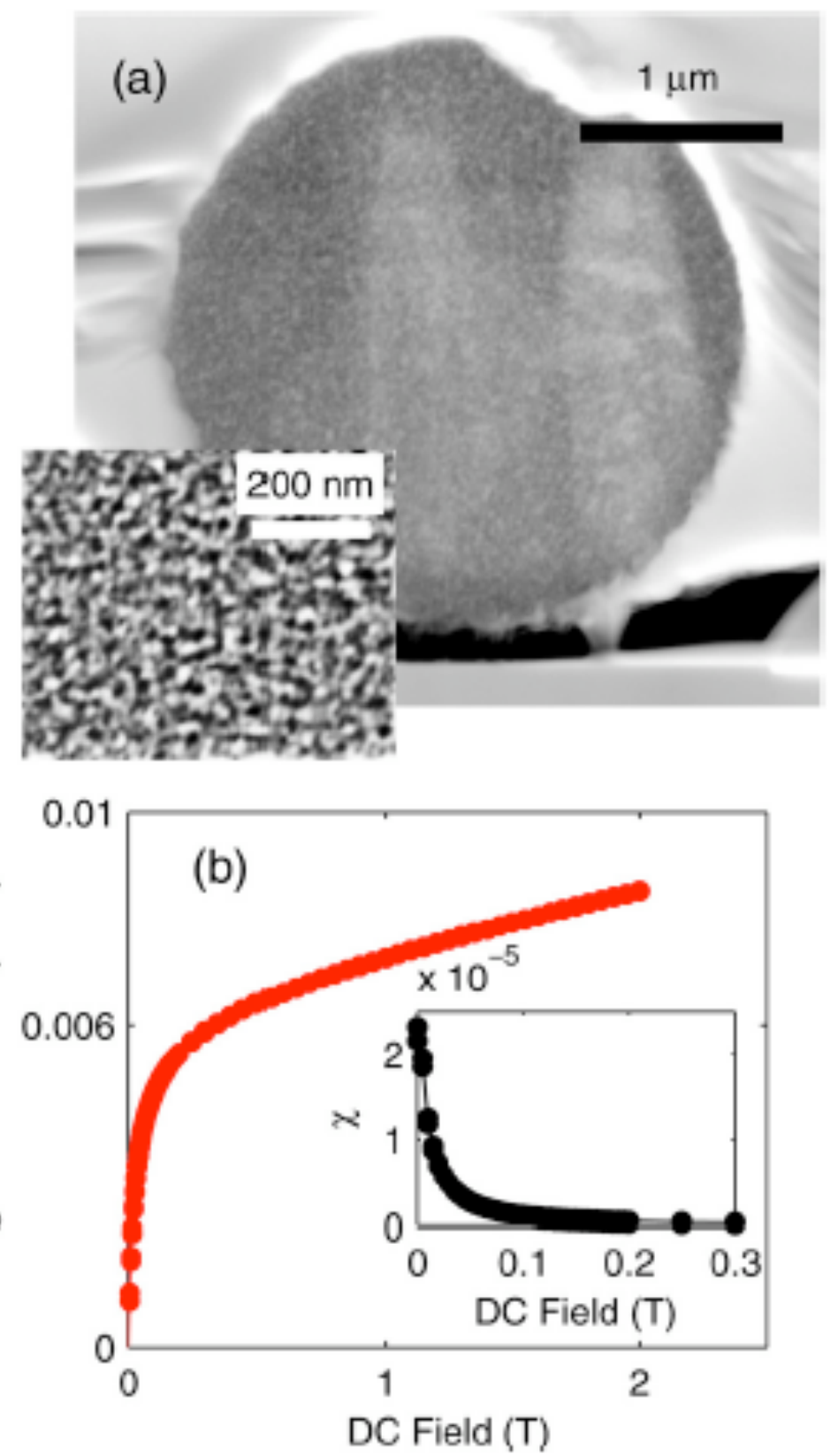

FIG. 1. (a) Dark field TEM image of a $100 \mathrm{~nm}$ thick foil made of $2.7 \mathrm{~lm}$ Dynal bead. Ferrite nanoparticles appear as bright spots on the dark background of the polymer matrix. Inset: Magnified section of the bead in which the nanoparticle contrast has been manually enhanced. (b) Measurements of the magnetic moment on a large ensemble of $1 \mathrm{~lm}$ Dynal beads at room temperature. The inset shows the field dependence of the magnetic susceptibility. 
The parameters configured to move the bead were as follows: the $\mathrm{XY}$ velocity. $2 \mathrm{~lm}=\mathrm{s}$, the $\mathrm{Z}$ distance._50 $\mathrm{nm}$, and the $\mathrm{Z}$ velocity. $25 \mathrm{~nm}=\mathrm{s}$. The $\mathrm{XY}$ velocity is the tip's lateral speed when moving as a nanomanipulator in real time, the $\mathrm{Z}$ distance is the distance when the tip is pushed into the sample surface, and the $Z$ velocity is the vertical speed of the tip as it presses into the sample surface. In the third step, the area was imaged after each nanomanipulation step in order to confirm the resulting position of the beads. The AFM nanomanipulation steps were repeated a few times, as depicted in Fig. 2, until only one bead remained on the Hall sensor while all other beads were pushed away (Fig. 2(d)). The AFM nanomanipulation technique is totally non-invasive and preserves good electronic properties of the Hall sensors.

\section{Measurement setup}

Bead detection was carried out using an ac-dc detection scheme modified with a phase control setup.6,12 A dc magnetic field BDC, generated by an electromagnet, was applied in the direction normal to the sample surface, together with an ac magnetic field BAC with a frequency f. $210 \mathrm{~Hz}$ generated by an inductive coil (L . 5:87 mH, R . 16:1 X). The sample was held at a distance of a few millimeters above the coil, where the peak-to-peak ac field amplitude was $9.2 \mathrm{mT}$ for $\mathrm{f}<500 \mathrm{~Hz}$. An amplitude drop due to coil capacitive effects was observed at higher frequencies. A number of precautionary measures were undertaken in order to create a "metal-free" environment in the close vicinity of the Hall sensor. Both the sensor and the coil were enclosed in a plastic breakout box held within the electromagnet gap by plastic spacers. Nylon screws were used where possible in order to avoid eddy currents in the near vicinity of the sample, and all unnecessary metallic material was removed.

The double cross was biased by a battery driven dc current source with Ibias. 1 1A, and the first harmonic ac voltage signal was measured between the transversal leads simultaneously on both crosses through two SR830 lock-in amplifiers using the voltage drop across the ac coil as a signal reference for both the frequency and the phase. 12 


\section{CHARACTERIZATION OF HALL SENSOR}

InSb double crosses have been fully characterized. The Hall coefficients $\left(\mathrm{RH}_{\mathrm{H}}\right)$ were measured using bias currents between 0.1 and $5 \mathrm{lA}$ and dc magnetic fields of up to $0.4 \mathrm{~T}$. The best $\mathrm{RH}_{\mathrm{H}}$ value measured for this type of device is ${ }_{-} 1100 \mathrm{X}=\mathrm{T}$ (Fig. 3(a)). The noise power spectral density was measured in the frequency range of $1 \mathrm{~Hz}$ to $12 \mathrm{kHz}$ and bias currents up to 5 lA, directly connecting a SR770 FFT spectrum analyzer to the voltage leads. The best white noise level was _ $9 \mathrm{nV}=\operatorname{root} \mathrm{Hz}$ at $\mathrm{f}>130 \mathrm{~Hz}$. Below this frequency, the characteristic $1=\mathrm{f}$ behavior was observed. The position of the corner between $1=\mathrm{f}$ and the white noise spectrum was found to increase with the bias current, whereas the level of the white noise was independent of the bias current and defined only by the resistance of the leads, $S_{\mathrm{wn}} .4 \mathrm{kBTR}$ (Fig. 3(b)).
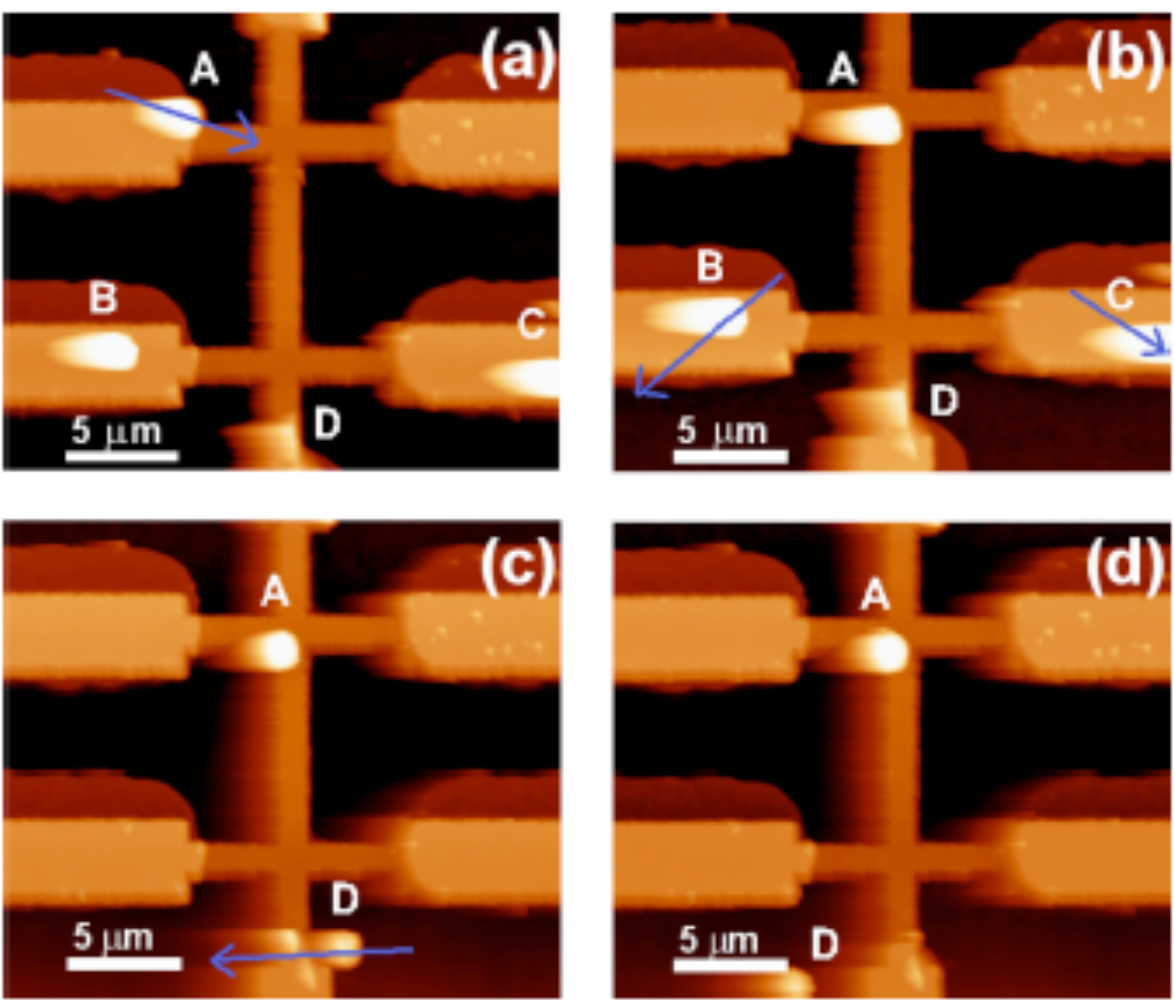

FIG. 2. AFM images of the double-cross Hall sensor with Dynal beads. The scanned area is $25 \_25$ lm2. (a) Initial configuration of four beads (A, B, C, and D) on the sensor. (b) Bead A has been moved into the final position, whereas the other beads (B, C, and D) are in their original positions. (c) Beads B and C have been removed from the horizontal arms of the sensor. (d) Bead D has been removed, and bead A remains in the final configuration on the active part of the top sensor. The blurred contrast of the bead is due to the high rate of the AFM imaging, 
which is necessary in order to minimize further interaction between the probe and the bead. The arrows indicate the directions of the bead movements.
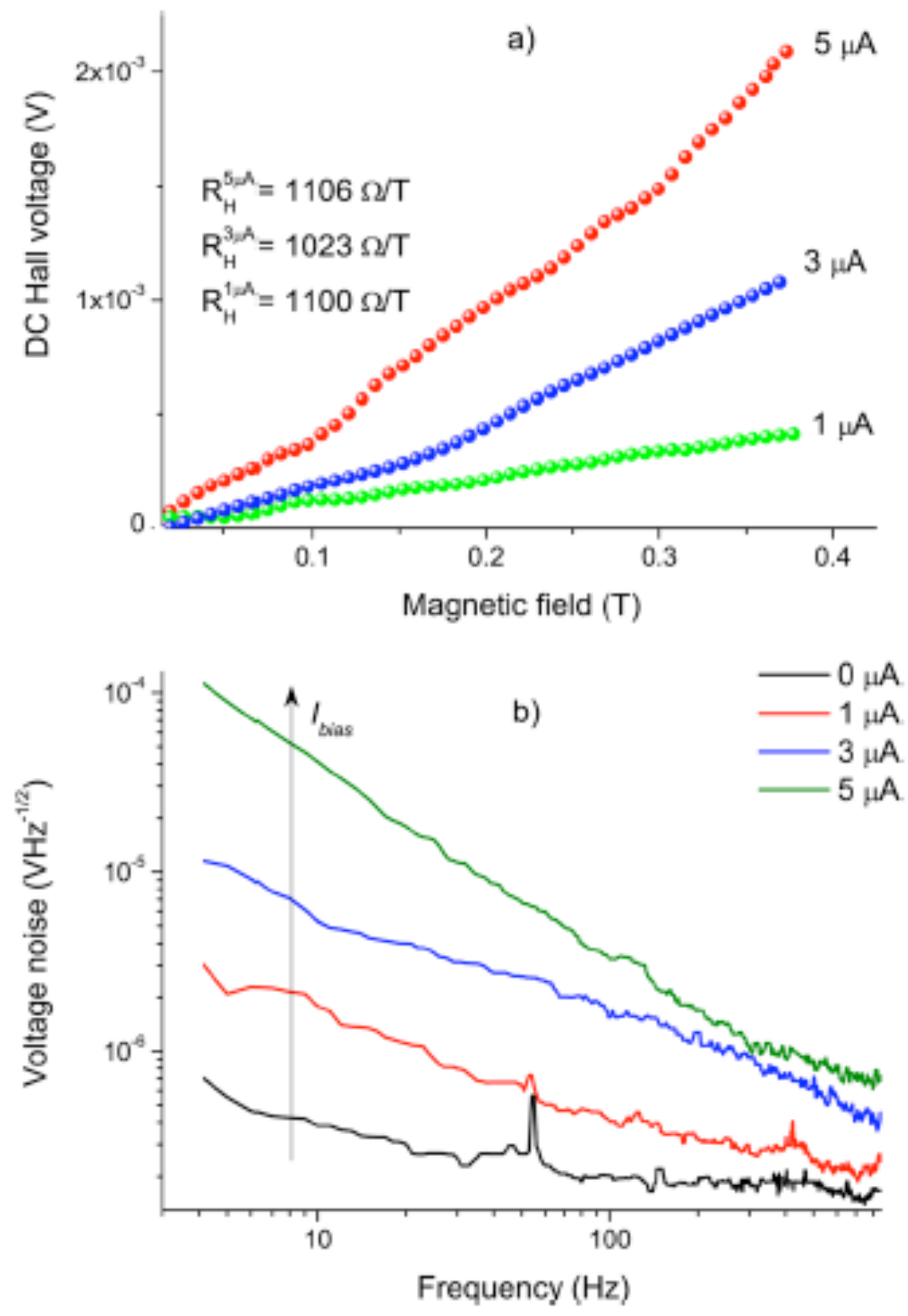

FIG. 3. (a) dc Hall coefficient and (b) noise power spectral density measurements for a $1 \mathrm{~lm}$ device at different bias currents. 


\section{AC-DC DETECTION METHOD}

The ac Hall voltage measured in the presence of a bead of susceptibility $v$ is given by 12

$$
V_{\mathrm{H}}^{\mathrm{AC}}\left(B_{\mathrm{DC}}\right)=I_{\text {bias }} R_{\mathrm{H}}\left[1+C \chi\left(B_{\mathrm{DC}}\right)\right] B_{\mathrm{AC}},
$$

where $\mathrm{C}$ is a geometrical parameter describing the coupling between the bead and the sensor. $\mathrm{V}_{\mathrm{AC}} \mathrm{H}$ is in phase with $\mathrm{BAC}$, and thus it has a constant $\mathrm{p}=2$ phase shift with respect to the voltage drop across the coil, which constitutes the lock-in phase reference. As demonstrated in our previous work,12 an inductive signal Vind, generated by the unavoidable coupling between voltage circuits' open loops and varying in time magnetic fields, is always present in the current measurement setup. However, Vind must be proportional to the time derivative of $\mathrm{BAC}$, which has a phase shift of $\mathrm{p}=2$ with respect to Taking BAC as a reference, phase sensitive measurements allow us to separate a real bead signal in the in-phase signal $V_{x}$ from inductive effects:

$$
V_{\mathrm{x}}=I_{\text {bias }} R_{\mathrm{H}}[1+C \chi] B_{1},
$$

where $\mathrm{B}_{1}$ is the ac field amplitude. The phase of the ac signal is affected only by parasitic inductive effects and could be used as a control parameter during particle detection experiments. The out of phase component $\mathrm{V}_{\mathrm{y}}$ is influenced by inductive pick-ups and parasitic ferromagnetic signals. In principle, assuming the presence of some practically unavoidable ferromagnetic materials in the near vicinity of the sample, a term Cferrovferro must be added within the square brackets in Eq. (2). This contribution is physically analogous to that of the bead under measurement, but it could be heavily reduced by working with relatively low dc magnetic fields $\left(\mathrm{BDC}_{-}{ }_{0.1}\right)_{12}$ The chosen ac frequency, $\mathrm{f}$. $210 \mathrm{~Hz}$, ensures a reasonably low noise level along with a clear separation of the inductive and real bead signals. Working with higher frequencies would allow a better signal-to-noise ratio. However, at $\mathrm{f}>400 \mathrm{~Hz}$, we observed a phase shift in the lock-in reference signal due to the capacitance of the ac coil, i.e., the voltage drop across the coil is no longer in a well-defined phase relation with respect to BAc. This effect leads to an incomplete separation of the bead signal in $V_{x}$ and, therefore, limits the working frequency range. In the next section we present two independent experiments (i.e., when the dc field is 
changed in a step-wise or sweeping mode) demonstrating the detection of a $1 \mathrm{~lm}$ superparamagnetic Dynal bead using a phase control ac-dc method. For a complete analysis of the phase sensitive detection method and the parasitic signals observed in our setup, the reader is referred to our previous work.12

\section{RESULTS}

The topography of the sample and the process of nanomanipulation (AFM pushing) are shown in Fig. 2. AFM measurements confirm a $1 \mathrm{~lm}$ size of the bead as specified by the supplier (Fig. 1, left inset). The panels of Fig. 2 demonstrate the steps of the movements of four beads (labeled A-D), where bead A is being moved toward the central part of the top cross and all other beads are pushed away from the active parts of the sensor. The blue arrows indicate the directions and movement paths of all beads. Figure 2 (a) shows the initial configuration of the beads on the sensor after deposition. In Fig. 2(b), bead A has been moved into its final position, whereas the other beads (B, C, and D) are still in their original positions. In Fig. 2(c), beads B and C have been removed from the horizontal arms of the sensor, whereas bead $D$ is still in its original position. Finally, in Fig. 2(d) bead D has been removed, and bead A remains on the top Hall sensor. The blurred contrast of the bead is due to the high rate of the AFM imaging, which is necessary in order to minimize further interaction between the probe and the bead.

\section{A. dc steps}

Rapid particle detection was achieved by applying a step-wise dc field that changed rapidly between two levels, $\mathrm{B}_{0} \mathrm{DC}$ (step duration.60 s) and B1 DC (step duration.30 s). In the presence of a bead, step-wise signals are expected to be measured in $V_{x}$ with an amplitude derivable from Eq. (2):

$$
V_{\mathrm{x}}\left(B_{\mathrm{DC}}^{1}\right)-V_{\mathrm{x}}\left(B_{\mathrm{DC}}^{0}\right)=I_{\mathrm{bias}} R_{\mathrm{H}} C\left[\chi\left(B_{\mathrm{DC}}^{1}\right)-\chi\left(B_{\mathrm{DC}}^{0}\right)\right] .
$$

Values of $\mathrm{B}_{0} \mathrm{DC} .0 \mathrm{~T}$ and $\mathrm{B}_{1} \mathrm{DC} .0: 1 \mathrm{~T}$ were used in the experiment. A relatively low $\mathrm{B}_{1}$ DC was adopted in order to reduce parasitic magnetic effects due to unwanted ferromagnetic material in the surroundings of the sample. However, the large decrease 
measured in v.BDC. between 0 and $0.1 \mathrm{~T}$ (see the inset in Fig. 1(b) and Eq. (3)) allowed an appreciable step in the amplitude of the response signal. Figure 4 shows a comparison between the in-phase $\left(V_{x}\right)$ and out-of-phase $\left(V_{y}\right)$ signals from the empty cross (Fig. $4(a)$ ) and the cross equipped with a bead (Fig. 4(b)). In order to get a better comparison, raw $V_{x}$ and $V_{y}$ data were normalized with respect to $R_{H}$. In the presence of the bead, the signal shows negative steps with an amplitude of $\_500 \mathrm{nV}$, in agreement with Eq. (3) and Fig. 1(b) inset, considering that v.B0 DC. _v.B1 DC.. On the same cross, Vy shows smaller positive steps $\left(\_150 \mathrm{nV}\right)$. The control (empty) device showed an identical response (_ $200 \mathrm{nV}$ steps) on both $V_{x}$ and $V_{y}$ components, demonstrating that the difference observed in the first case is ascribable to the bead's presence. The step-wise response in $V_{y}$ is mainly due to parasitic inductive effects, whereas the $V_{x}$ steps on the empty cross can be attributed to the unavoidable presence of ferromagnetic material in the close vicinity of the device. In both cases, an incomplete separation of inductive and magnetic material effects could play a role. Thus, the step-wise method equipped with the phase control demonstrates direct proof of the presence $=$ absence of the magnetic bead on the sensor, which is essential for biomedical applications.

B. dc sweeps

The direct measurement of the susceptibility curve for a single bead could be performed by applying a sweeping dc field. Taking the difference between in-phase signals of crosses with and without the bead and normalizing with respect to the Hall coefficients, we obtain

$$
C \chi\left(B_{\mathrm{DC}}\right)=\frac{1}{I_{\text {bias }}}\left[\frac{V_{\mathrm{x}}^{1}}{R_{\mathrm{H}}^{1}}-\frac{V_{\mathrm{x}}^{2}}{R_{\mathrm{H}}^{2}}\right] \text {, }
$$

where the cross with a bead is labeled as 1 . The coupling constant, C.0.09, was numerically computed following Ref. 13. In Fig. 5 we show the experimental data (dots) worked out following Eq. (4). The fitting curve for the susceptibility is the first derivative of the Langevin function describing the magnetization of a system of N noninteracting magnetic moments lnano, i.e., nanosized ferrite particles comprising the Dynal bead. 


$$
\chi=\frac{N \mu_{\text {nano }}}{k_{\mathrm{B}} T}\left(\frac{k_{\mathrm{B}} T}{\mu_{\text {nano }} B_{\mathrm{DC}}}\right)^{2}-\frac{N \mu_{\mathrm{nano}}}{k_{\mathrm{B}} T} \frac{1}{\sinh ^{2}\left(\mu_{\operatorname{mano}} B_{\mathrm{DC}} / k_{\mathrm{B}} T\right)} .
$$

The fitted parameter lnano _ 430 lв is a reasonable estimation of the magnetic moment of one of the ferrite nanoparticles contained in a bead. Using our estimation of the number of nanoparticles per bead, we get a total magnetic moment $4_{-} 108 \mathrm{lB}$, which is only slightly larger than the magnetic moment of the $140 \mathrm{~nm}$ FePt bead detected in our previous work12 (_108 اв). This is compatible with the low nanoparticle density of the Dynal beads. It should be noted, however, that the main uncertainty here is in the accurate estimation of the number of ferrite nanoparticles per bead. Following Eqs. (3) and (4), we can compare the results of step and sweeping field experiments. Figure 5 shows a difference of _330 nV in $\mathrm{Cv}$ as BDC changes between 0 and $0.1 \mathrm{~T}$. This value must be compared with the difference observed in the $V_{x}$ step amplitudes between crosses with and without the bead, i.e., _350 nV, in good agreement with the sweeping experiment. The comparison between the shape of the single bead susceptibility curve (Fig. 5) and the same quantity measured for a large ensemble of beads (Fig. 1(b), inset) demonstrates a rather different behavior in low magnetic fields. This difference could be ascribed to dipolar bead-to-bead interaction, which possibly plays a significant role in the latter case. In a recent work by Aledealat et al.15 it was demonstrated that, depending on the position of the bead on the sensor, the Hall voltage output could change its sign. That is, if the bead was located roughly at the center of the cross, a conventional decrease of the dc Hall voltage was measured. However, if the bead was placed on one of the sensor arms, an opposite sign of the signal, i.e., an increase of the Hall voltage of a smaller amplitude, was observed. The sensitivity radius of the Hall cross was further defined based on the stray magnetic field associated with the bead and the noise level of the sensor. These very interesting results are, nevertheless, not applicable in the present case, as all "extra" beads were moved far away from the active area of the devices (i.e., more than $20 \mathrm{~lm}$ away along all arms). It should be noted, however, that the measurements in Ref. 15 were performed without a careful separation of in-phase and out-of-phase signals, which should further facilitate the interpretation of the experimental results. 
(a)

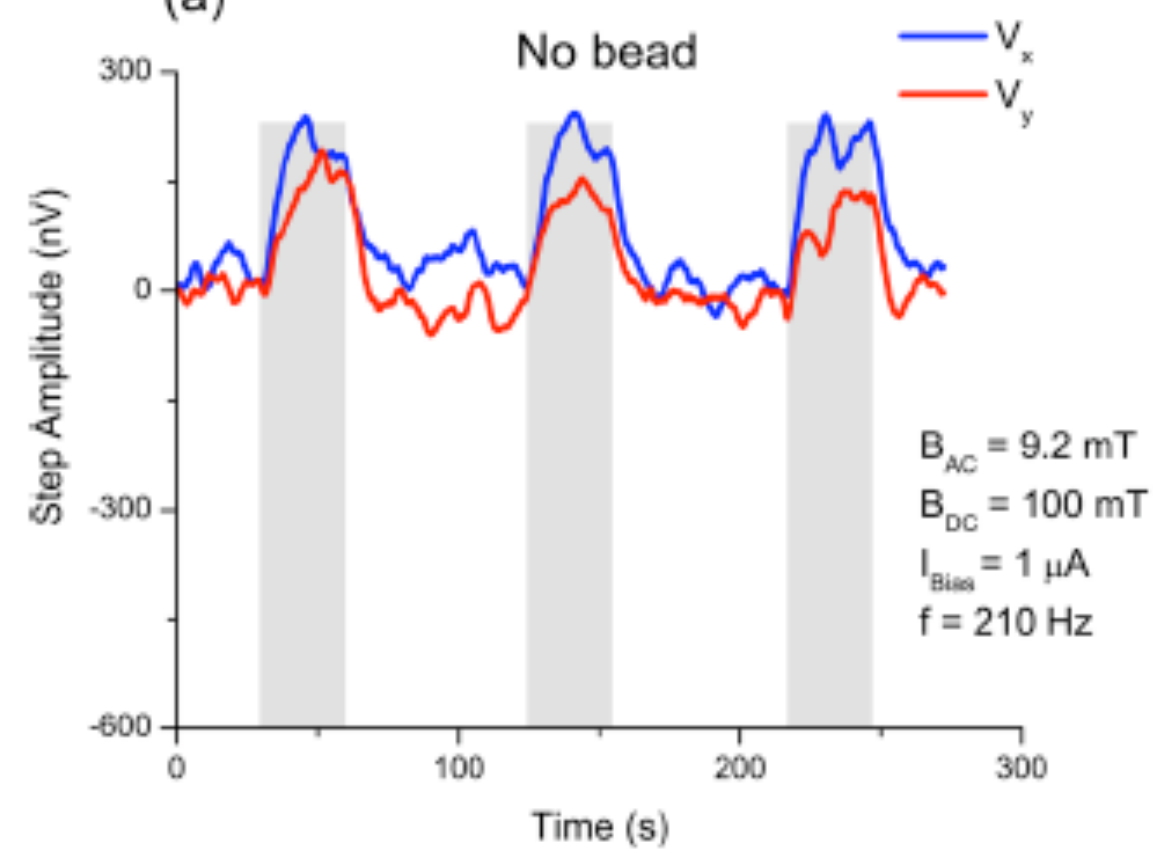

(b)

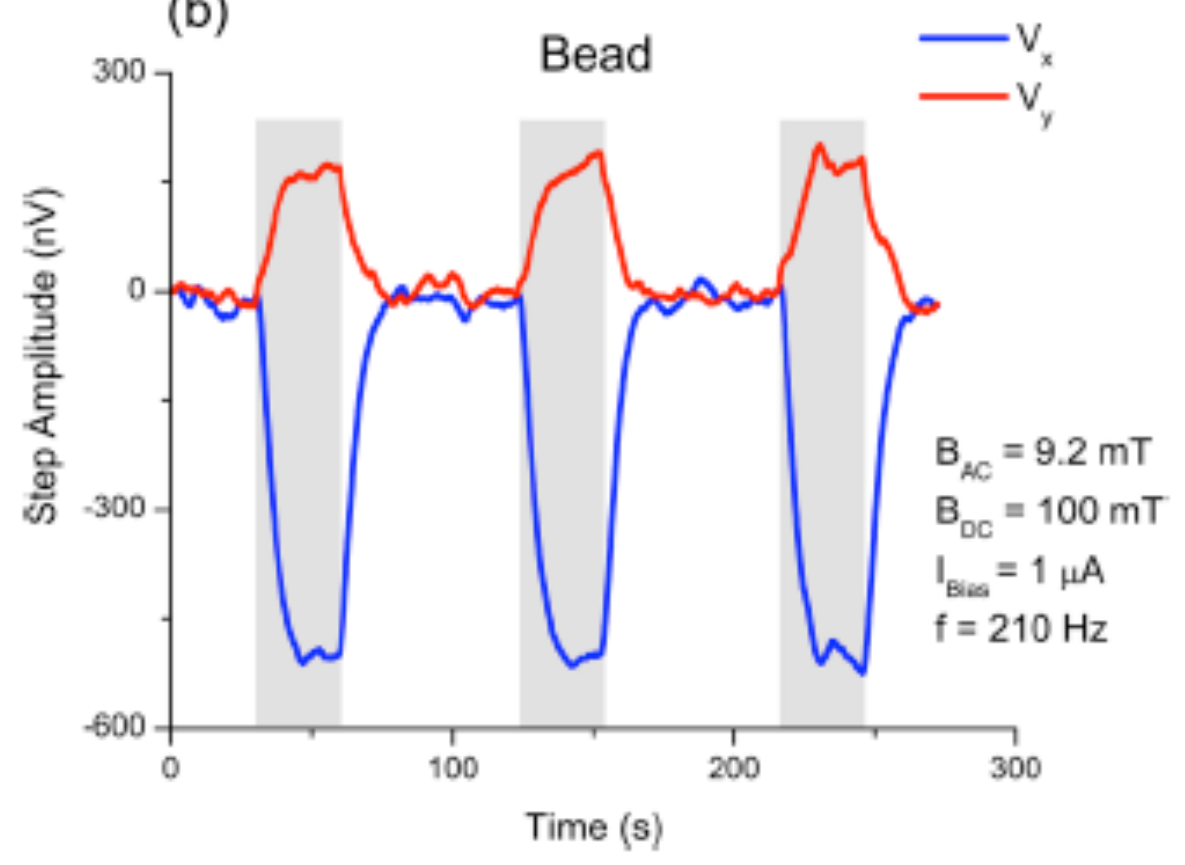

FIG. 4. (Color online) In-phase $\left(V_{x}\right)$ and out-of-phase $\left(V_{y}\right)$ components of the ac Hall voltage in response to BDC steps (BDc. $100 \mathrm{mT}$ ) with a duration of $30 \mathrm{~s}$ as measured on (a) an empty device and (b) a device with a Dynal bead. Note that the $V_{y}$ component always shows an increase of the voltage independent of the presence of the bead. The effect is associated with dominating inductive and parasitic ferromagnetic signals. The grey rectangles represent the state when BDC is on. 


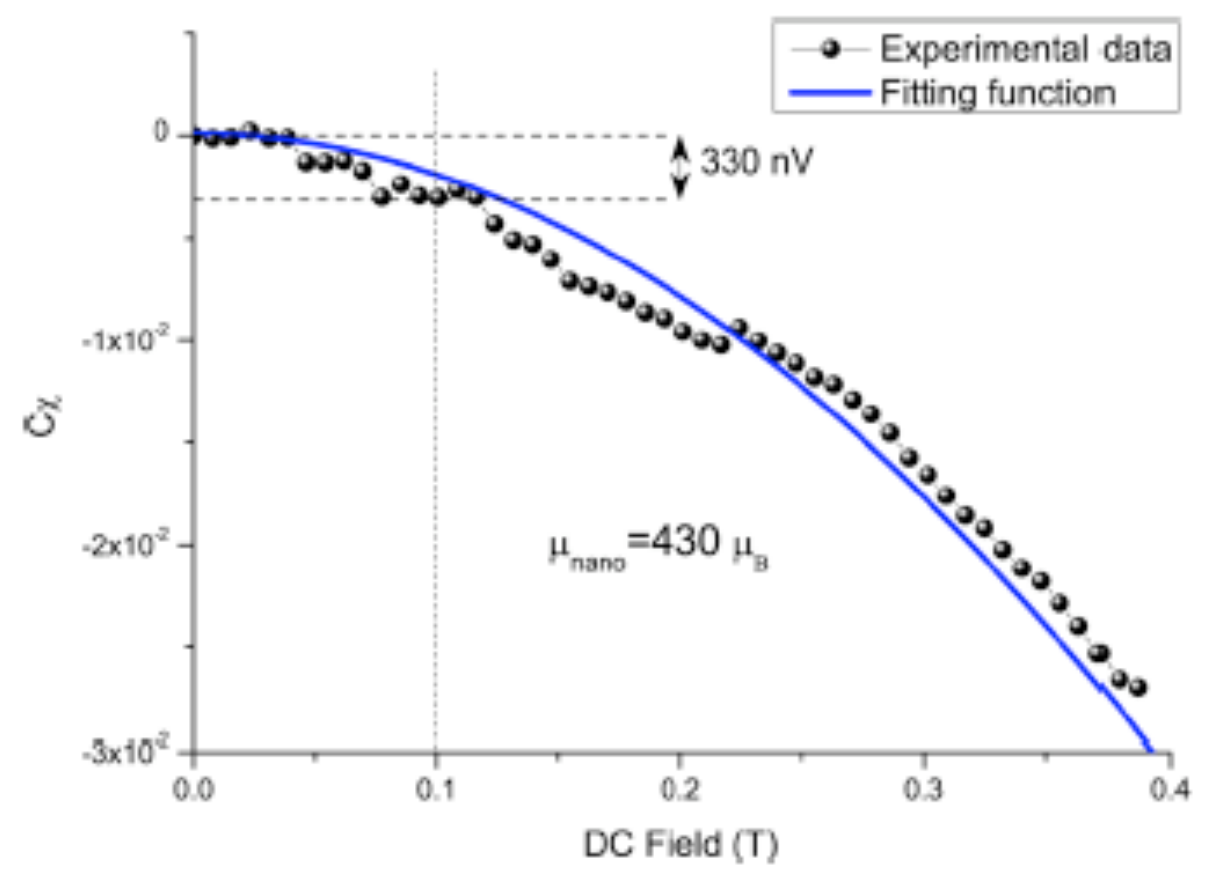

FIG. 5. (Color online) Product of the experimental magnetic susceptibility of a single Dynal bead and the bead-sensor coupling constant $C$ (dots) fitted by Eq. (5) (solid line). The dashed lines show the decrease in the $V_{x}$ component as BDC changes from 0 to $100 \mathrm{mT}$ (i.e., the amplitude of the dc-field step in Fig. 4(b)).

\section{CONCLUSIONS}

We present detection and susceptibility measurements of a single magnetic bead (Dynal, MyOne) with a diameter of $1 \mathrm{~lm}$ and a magnetic moment of _ $4_{-} 108 \mathrm{~B}$. The accurate particle positioning, achieved via non-invasive AFM nanomanipulation, allowed a significant improvement of the bead-to-sensor coupling. Very clear evidence of the bead's presence is demonstrated using a step-wise change of the dc field. The method provides direct proof of the magnetic bead's presence=absence on the sensor, which is necessary for numerous biological applications. The experimental procedure described here allows a clear and unambiguous separation of the real magnetic contribution due to the presence of the bead from inductive and other parasitic signals attributed to the measurement setup. Furthermore, using an alternative measurement technique (sweeping dc field) and taking the normalized difference between the in-phase signals of crosses with and without the bead, we demonstrate the direct measurement of the susceptibility curve for a single bead. The values of the ac Hall voltage obtained with these two experimental methods are in very good quantitative agreement. Thus, the method presented here opens up new possibilities 
for the reliable and accurate detection of small magnetic moments, which is of high importance for highly sensitive biological, medical, and environmental detectors, as well as for nanoscale metrological applications.

\section{ACKNOWLEDGMENTS}

This work has been funded by the UK BIS under Project No. 114503 and EU FP7 NanoSpin (T4.J02).

1Frost \& Sullivan, “World magnetic sensors markets,” Report N775-3, May 2010.

${ }_{2}$ K. Togawa, H. Sanbonsugi, A. Sandhu, M. Abe, H. Narimatsu, K. Nishio, and H. Handa, J. Appl. Phys. 99, $08 \mathrm{P} 103$ (2006).

${ }_{3}$ P. Manandhar, K.-S. Chen, K. Aledealat, G. Mihajlovic, C. S. Yun, M. Field, G. J. Sullivan, G. F. Strouse, P. B. Chase, S. von Molnar, and P. Xiong, Nanotechnology 20, 355501 (2009).

4A. Sandhu, H. Handa, and M. Abe, Nanotechnology 21, 442001 (2010).

${ }_{5}$ K. Vervaeke, E. Simoen, G. Borghs, and V. V. Moshchalkov, Rev. Sci. Instrum. 80, 074701 (2009).

${ }_{6}$ P. Besse, G. Boero, M. Demierre, V. Pott, and R. Popovic, Appl. Phys. Lett. 80, 4199 (2002).

7G. Landry, M. Miller, B. Bennett, M. Johnson, and V. Smolyaninova, Appl. Phys. Lett. 85, 4693 (2004).

${ }_{8}$ G. Mihajlovic, P. Xiong, S. von Molnar, K. Ohtani, H. Ohno, M. Field, and G. Sullivan, Appl. Phys. Lett. 87, $112502(2005)$.

${ }_{9}$ O. Florescu, M. Mattmann, and B. Boser, J. Appl. Phys. 103, 046101 (2008).

${ }_{10}$ O. Kazakova, J. C. Gallop, P. See, D. Cox, G. K. Perkins, J. D. Moore, and L. F. Cohen, IEEE Trans. Magn. 45, 4499 (2009).

${ }_{11}$ O. Kazakova, V. Panchal, J. Gallop, P. See, D. C. Cox, M. Spasova, and L. F. Cohen, J. Appl. Phys. 107, $09 \mathrm{E} 708(2010)$.

12L. Di Michele, C. Shelly, J. Gallop, and O. Kazakova, J. Appl. Phys. 108, 103918 (2010).

${ }_{13}$ G. Mihajlovic, K. Aledealat, P. Xiong, S. Von Molnar, M. Field, and G. J. Sullivan, Appl. Phys. Lett. 91, 172518 (2007).

14T. Zhang, S. Clowes, M. Debnath, A. Bennett, C. Roberts, J. Harris, R. Stradling, L. Cohen, T. Lyford, and P. Fewster, Appl. Phys. Lett. 84, 4463 (2004).

15K. Aledealat, G. Mihajlovic, K. Chen, M. Field, G. J. Sullivan, P. Xiong, P. B. Chase, and S. von Molnar, J. Magn. Magn. Mater. 322, L69 (2010). 